\title{
Noncontrast vestibular schwannoma surveillance imaging including an MR cisternographic sequence: is there a need for postcontrast imaging?
}

\author{
Karen Buch, MD, ${ }^{1}$ Amy Juliano, MD, ${ }^{2}$ Konstantina M. Stankovic, MD, PhD, ${ }^{3}$ Hugh D. Curtin, MD, ${ }^{2}$ \\ and Mary Beth Cunnane, MD²
}

1Department of Neuroradiology, Massachusetts General Hospital; and Departments of ${ }^{2}$ Radiology and ${ }^{3}$ Otolaryngology, Massachusetts Eye \& Ear Infirmary, Boston, Massachusetts

\begin{abstract}
OBJECTIVE The purpose of this study was to evaluate the use of a noncontrast MRI protocol that includes a cisternographic sequence (CISS/FIESTA/3D DRIVE) compared to a protocol that includes a gadolinium-enhanced sequence in order to determine whether a noncontrast approach could be utilized to follow vestibular schwannomas.

METHODS A total of 251 patients with vestibular schwannomas who underwent MRI of the temporal bones that included both cisternographic sequence and postcontrast T1 imaging between January 2000 and January 2016 for surveillance were included in this retrospective study. The size of the vestibular schwannomas was independently assessed on a noncontrast MR cisternographic sequence and compared to size measurements on a postcontrast sequence. The evaluation of intralesional cystic components (identified as T2 signal hyperintensity) and hemorrhagic components (identified with intrinsic T1 hyperintensity) on noncontrast MR sequences was compared to evaluation on postcontrast MR sequences to determine whether additional information could be derived from the postcontrast sequences. Additionally, any potentially clinically significant, incidentally detected findings on the postcontrast $\mathrm{T} 1$ sequences were documented and compared with the detection of these findings on the precontrast images.
\end{abstract}

RESULTS No significant difference in vestibular schwannoma size was found when comparing measurements made on the images obtained with the MR cisternographic sequence and those made on images obtained with the postcontrast sequence $(p=0.99)$. Noncontrast MR images were better (detection rate of $87 \%$ ) than postcontrast images for detection of cystic components. Noncontrast MR images were also better for identifying hemorrhagic components. No additional clinically relevant information regarding the tumors was identified on the postcontrast sequences.

CONCLUSIONS Based on the results of this study, a noncontrast MR protocol that includes a cisternographic sequence would be sufficient for the accurate characterization of size and signal characteristics of vestibular schwannomas, obviating the need for gadolinium contrast administration for the routine surveillance of these lesions.

https://thejns.org/doi/abs/10.3171/2018.3.JNS1866

KEYWORDS vestibular schwannoma; internal auditory canal; MRI

$\mathrm{M}$ AGNETIC resonance cisternographic images, such as CISS, FIESTA, or 3D DRIVE, provide a heavily fluid-weighted sequence with high spatial resolution. These attributes make the cisternographic sequence advantageous for use in temporal bone imaging, particularly when assessing neural elements within the internal auditory canal. Prior temporal bone imaging studies have demonstrated a high sensitivity of the cisternographic sequence for the detection of vestibular schwannomas ranging in size between 2 and $20 \mathrm{~mm} .^{1,15,19}$ To date, these studies have all been performed in relatively small patient cohorts. ${ }^{1,15,19}$

In the radiology literature, the use of linear gadoliniumbased MRI contrast agents has been questioned, with recent data demonstrating the accumulation of gadolinium-based contrast agents in the brain parenchyma. ${ }^{10,11,14,21}$ The results of these studies have caused physicians to give additional consideration to the necessity of intravenous contrast use. This is of particular concern in patients who undergo frequent MRI examinations for surveillance imaging, such as 


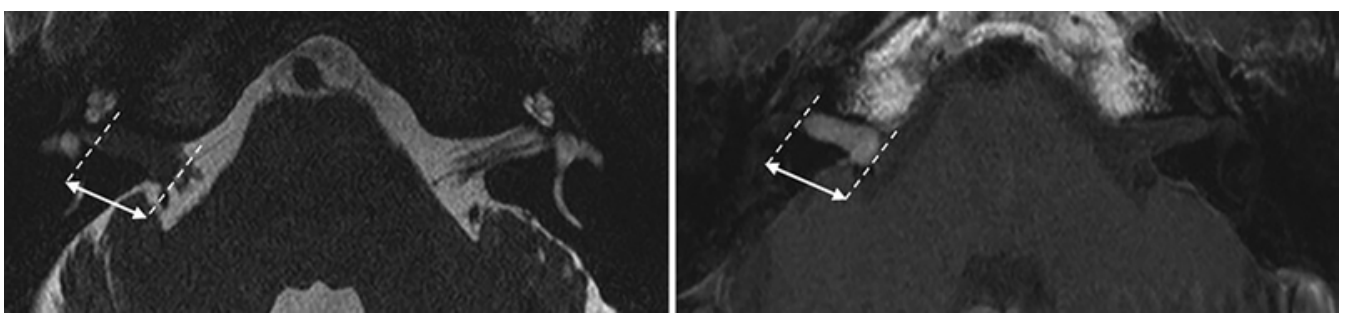

FIG. 1. Example of the long-axis measurement of a vestibular schwannoma on an MR cisternographic sequence (left) and a T1 postcontrast image (right) obtained in a 38-year-old woman with a right vestibular schwannoma.

patients with vestibular schwannomas. ${ }^{6,7,9,23}$ Patients with these tumors typically undergo annual or biannual contrast-enhanced MRI for surveillance, leading to a higher exposure to gadolinium contrast. .,7,9,23 $^{-1}$

The purpose of this study was to determine whether a contrast-enhanced MRI protocol is more beneficial than a noncontrast MRI protocol that includes an MR cisternographic sequence in a large population of patients with vestibular schwannoma. Specifically, we sought to evaluate whether a conventional contrast-enhanced MRI protocol has any advantage compared to the noncontrast protocol for evaluating vestibular schwannoma size and intralesional characteristics such as cystic components or hemorrhage. In addition, we attempted to determine whether any potentially clinically significant incidental findings that would be identified with a contrast-enhanced MRI protocol might be missed with a noncontrast protocol.

\section{Methods \\ Patient Cohort}

This was a retrospective, IRB-approved study performed at a tertiary medical center (Massachusetts Eye \& Ear Infirmary). A total of 278 patients with a diagnosis of vestibular schwannoma who underwent a temporal bone MRI examination with both a noncontrast, cisternographic and a conventional T1 postcontrast sequence between January 2000 and January 2016 were included in this study. This included a cohort previously described, with data collected before 2012, as well as the results of a search of our picture archiving and communication system (PACS) between 2012 and 2016 for patients with known diagnosis of vestibular schwannoma who had sequential scans that included postcontrast imaging as well as a cisternographic sequence. ${ }^{13}$ Exclusion criteria included prior partial or complete resection of the vestibular schwannoma and motion or technical artifacts precluding a diagnostic assessment of the images. A total of 27 studies were excluded based on the aforementioned criteria, leading to the inclusion of 251 patients in the analysis. Basic patient descriptors, including age and sex, were recorded.

\section{MRI Scanning Parameters}

All scans performed prior to December 2006 were performed on a 1.5-T Siemens scanner. Sequences performed included a sagittal spin echo T1-weighted sequence (TR/ TE 500/12 msec, NEX 1, matrix $210 \times 512$, FOV $23 \times 29.5$ cm), axial T2 (TR/TE 4032/90 msec, NEX 1, matrix 21 $\times 27 \mathrm{~cm}$ ), axial FLAIR (TR/TE 9999/110 msec, NEX 1, matrix $198 \times 512$, FOV $21 \times 27 \mathrm{~cm}$ ), dedicated pre- and postcontrast T1-weighted axial images through the temporal bone $(\mathrm{TR} / \mathrm{TE} 500 / 12 \mathrm{msec}$, NEX 2, matrix $192 \times 512$, FOV $18 \times 23.1 \mathrm{~cm})$, dedicated coronal postcontrast images through the temporal bone (TR/TE 450/15 msec, NEX 3, matrix $192 \times 512$, FOV $17 \times 21.8)$, and a precontrast isotropic cisternographic image (TR/TE 12.25/5.9 msec, NEX 1 , matrix $230 \times 1024$, FOV $20 \times 25.7$ ).

All scans performed after December 2006 were performed on a 3-T Philips scanner. Sequences performed included a sagittal spin echo T1-weighted sequence (TR/ TE 450/10 msec, NEX 1, matrix $272 \times 271$, FOV $37 \times 24.4$ $\mathrm{cm})$, axial T2 (TR/TE 3366/80 msec, NEX 3, matrix $210 \times$ 176, FOV $37 \times 24.4 \mathrm{~cm}$ ), axial FLAIR (TR/TE 11,000/125 msec, NEX 2, matrix $284 \times 206$, FOV $36.5 \times 24 \mathrm{~cm}$ ), axial diffusion-weighted images (TR/TE 3124.67/53.29 msec, NEX 2, matrix $116 \times 93$, FOV $36 \times 23.7 \mathrm{~cm})$, a precontrast isotropic cisternographic image (TR/TE 17,000/190 msec, NEX 3, matrix $412 \times 337$, FOV $23.3 \times 14$ ), and dedicated pre- and postcontrast T1-weighted images through the temporal bone (TR/TE 478.15/11 msec, NEX 3, matrix $312 \times 311$, FOV $24.3 \times 16 \mathrm{~cm})$.

\section{Image Analysis}

\section{Evaluation of Vestibular Schwannoma Size}

The images from each of the 251 cases were reviewed by a neuroradiology fellow, blinded to the patients' clinical history. For each patient, the MR cisternogram sequence and the postcontrast T1 sequence were obtained at the same time, and on the same scanner platform. The greatest transverse dimension of the vestibular schwannomas was measured on the noncontrast MRI sequences, specifically, on the MR cisternographic image. Six weeks following the initial evaluation on the noncontrast MR images, the greatest transverse dimension of the vestibular schwannoma was then measured only on the T1 postcontrast images (Fig. 1). This separation in time between the evaluation of the MR cisternogram and T1 postcontrast images was done to minimize bias. This methodology is similar to that used in the study by Ozgen et al. ${ }^{19}$

Interrater variability was calculated for measurements of vestibular schwannoma size by comparing measurements performed by a neuroradiology fellow with those done by a head and neck radiology attending. A subset of 30 cases of vestibular schwannomas was reviewed independently by each rater. As before, initially only the cisternographic images were reviewed, then only the T1 
TABLE 1. Comparison of vestibular schwannoma size and internal characteristics seen on cisternographic images versus conventional postcontrast images

\begin{tabular}{lccc}
\hline \multicolumn{1}{c}{ Parameter } & $\begin{array}{c}\text { Cisternogram } \\
\text { Image }\end{array}$ & $\begin{array}{c}\text { Postcontrast } \\
\text { Image }\end{array}$ & $\begin{array}{c}\text { Outcome } \\
\text { Measure }\end{array}$ \\
\hline Mean & $15.07 \mathrm{~mm}$ & $15.08 \mathrm{~mm}$ & $p=0.99$ \\
\hline Standard deviation & $8.66 \mathrm{~mm}$ & $8.71 \mathrm{~mm}$ & \\
\hline Minimum & $2 \mathrm{~mm}$ & $2 \mathrm{~mm}$ & \\
\hline Maximum & $48 \mathrm{~mm}$ & $47 \mathrm{~mm}$ & \\
\hline Cystic & $39 / 45$ & $12 / 45$ & $86.7 \%$ vs $26.7 \%$ \\
\hline Hemorrhage & $0 / 5$ & $0 / 5$ & $0 \%$ vs $0 \%$ \\
\hline
\end{tabular}

postcontrast image at least 1 week later. The greatest measured transverse dimension of the vestibular schwannomas was recorded. This subset of 30 cases was used to perform interrater reliability derived from a power calculation performed by a biostatistician.

\section{Evaluation of Vestibular Schwannoma Internal Characteristics}

The vestibular schwannomas were assessed for the presence of internal cystic components, conventionally identified by focal T2 hyperintensity within the tumor or at the tumor periphery, and intralesional hemorrhage, as demonstrated by $\mathrm{T} 1$ signal intensity shortening within the tumor.

These findings were assessed on the noncontrast MR images first; then 6 weeks later, they were assessed on the postcontrast images. These results were recorded separately.

The sensitivity of the cisternographic sequence alone for the detection of cystic and hemorrhagic components was then calculated and compared to the performance of the postcontrast T1-weighted sequence, using the imaging appearance of the vestibular schwannoma on the conventional precontrast T1-weighted sequence and T2-weighted sequences as an internal reference.

\section{Evaluation of Potentially Clinically Significant Incidental Findings}

The last component of the imaging analysis included an assessment of potentially clinically significant incidental findings. Each of the 251 patient MRI examinations was reviewed for the presence of incidental findings, which while not relevant to the management of the vestibular schwannoma, might direct the patient to additional clinical evaluation or management.

Initially, the noncontrast MRI sequences including the MR cisternographic sequence were reviewed together. Six weeks later, the postcontrast sequences were reviewed for the same assessment.

The presence and type of incidental finding was recorded for each round of review. Each of the incidental findings was then assigned a clinical significance score as follows: $0=$ no incidental findings, $1=$ incidental findings of no clinical significance, $2=$ incidental findings which require nonurgent follow-up, 3 = incidental findings which would require urgent/emergent management.

The number of incidental findings stratified by type and clinical significance score was recorded. Potentially clini- cally significant findings that could only be identified on postcontrast sequences were also noted.

\section{Results \\ Patient Demographics}

Of the 251 patients included in this study, 121 were male (48\%) and 130 were female (52\%). The patients' mean age was $54.7 \pm 8.2$ years. A total of 30 of these 251 patients $(12 \%)$ had a diagnosis of neurofibromatosis type 2 .

\section{Vestibular Schwannoma Size and Interrater Reliability}

On the cisternographic images, vestibular schwannomas ranged in size from 2 to $48 \mathrm{~mm}$, with an average size of $15.07 \pm 8.66 \mathrm{~mm}$, compared to $15.08 \pm 8.71 \mathrm{~mm}$ as measured on the postcontrast images $(\mathrm{p}=0.99)$ (Table 1$)$.

Interrater agreement for the evaluation of vestibular schwannoma size as measured on the MR cisternographic sequence was excellent $(\mathrm{k}=0.89)$. Interrater agreement for the evaluation of vestibular schwannoma size as measured on the conventional postcontrast images was also excellent $(\mathrm{k}=0.91)$. There was no statistically significant difference between vestibular schwannoma size measured on the MR cisternographic sequence and the size measured on the T1 postcontrast image $(\mathrm{p}=0.77)$.

\section{Evaluation of the Internal Components}

A total of 45 of 251 (18\%) vestibular schwannomas contained a presumed cystic component, ranging in size from 2 to $19 \mathrm{~mm}$. Internal cystic components were best identified on the conventional T2-weighted images. A direct comparison of the MR cisternographic sequence to the conventional T2-weighted images noted that 39 of 45 cystic components were seen on the MR cisternographic sequence $(86.7 \%)$.

When evaluating the presence of cystic components on the postcontrast T1-weighted images alone, only 11 cystic components were identified, compared to the 45 presumed cystic components seen on the conventional T2-weighted images, as well as 39 on the MR cisternographic sequence.

A total of 5 of the 251 vestibular schwannomas (0.02\%) demonstrated intrinsic T1 hyperintense components that were attributed to possible intralesional hemorrhage (Fig. 2 ). These presumed internal hemorrhagic components were only appreciated on the precontrast T1-weighted images, and could not be appreciated on the MR cisternographic sequences or the T1 postcontrast sequences.

\section{Evaluation of Incidental Findings}

Potentially clinically significant incidental findings were seen in a total of 80 patients $(32 \%)$ (Table 2). In the other 171 patients $(68 \%)$, there were no clinically significant incidental findings (grade 0). A clinical significance score of 1 was given to findings in a total of 64 patients (25\%), a score of 2 was given to findings in 8 patients $(3 \%)$, and a score of 3 was given to findings in 2 patients $(0.8 \%)$ (Table 2) (Figs. 1 and 2). Findings for which clinical significance scores of 1 were given included paranasal sinus disease in 34 patients, chronic white matter disease in 18 patients, arachnoid cysts in 5 patients, meningiomas in 3 

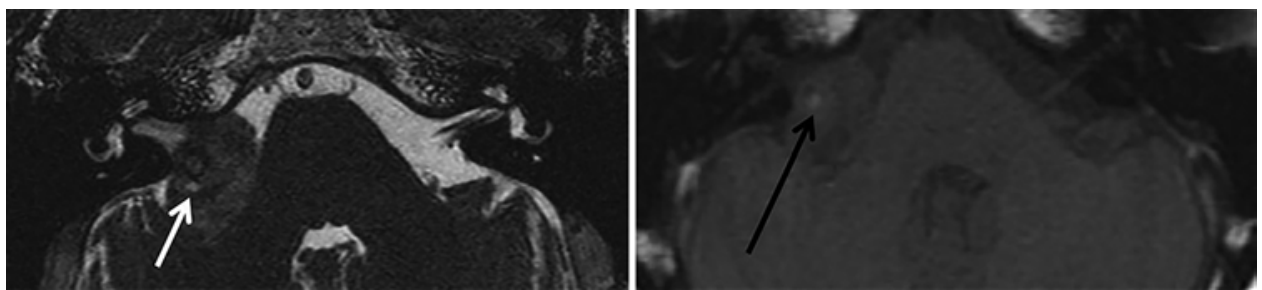

FIG. 2. Axial cisternographic image (left) and precontrast T1-weighted image (right) obtained in a 58-year-old woman with a right vestibular schwannoma. A focus of intrinsic T1 hyperintensity (black arrow) corresponding to presumed hemorrhage is not well identified on the cisternographic image. A cystic component (white arrow) is well visualized on the cisternographic image.

patients, Rathke's cleft cysts in 2 patients, and Tornwaldt cysts in 2 patients. A clinical significance score of 2 was given for pleomorphic parotid adenomas in 3 patients, a pituitary mass in 1 patient, a dural prostate cancer metastasis in 1 patient, labyrinthitis in 1 patient, and intracranial cavernous malformations 2 patients. A clinical significance score of 3 was given for the finding of demyelinating disease in 1 patient and acute-to-subacute pontine infarction in 1 patient (Fig. 3).

These potentially clinically significant incidental findings were identified on the review of the noncontrast sequences alone. There was no potentially clinically significant incidental finding identified on the postcontrast sequences that was not identified on the noncontrast MR sequences.

\section{Discussion}

The results of this study demonstrate that a noncontrast MRI protocol that includes an MR cisternographic sequence performs as well for the evaluation of vestibular schwannoma size as a conventional MRI protocol that includes postcontrast sequences. In addition, our analysis also showed that the noncontrast protocol performed as well as the conventional protocol for the detection of potentially clinically significant incidental findings. Moreover, the results of this study also demonstrated that intralesional characteristics of vestibular schwannomas, including cystic components and intralesional hemorrhage, are better assessed on the noncontrast MRI sequences. Based on these observations, the postcontrast imaging may not be needed in the setting of routine surveillance images for patients with vestibular schwannomas.

TABLE 2. Summary of incidental findings stratified by potential clinical significance

\begin{tabular}{ccc}
\hline $\begin{array}{c}\text { Clinical } \\
\text { Significance Score }\end{array}$ & \multicolumn{1}{c}{ Description } & $\begin{array}{c}\text { No. of } \\
\text { Patients }\end{array}$ \\
\hline 0 & No incidental findings & $171(68 \%)$ \\
\hline 1 & $\begin{array}{c}\text { Incidental findings with no clinical } \\
\text { significance }\end{array}$ & $64(25 \%)$ \\
\hline 2 & $\begin{array}{c}\text { Incidental findings that require } \\
\text { nonurgent clinical follow-up }\end{array}$ & $8(3 \%)$ \\
\hline 3 & $\begin{array}{c}\text { Incidental findings that require } \\
\text { urgent/emergent follow-up }\end{array}$ & $2(0.8 \%)$ \\
\hline
\end{tabular}

${ }^{*}$ Number of patients with incidental findings in the given clinical significance category.
The findings from this study support the findings of prior studies demonstrating the high accuracy of the cisternographic sequence for the detection of vestibular schwannoma and characterization of lesion size., ${ }^{1,15,19}$ This study builds on prior studies in demonstrating the low prevalence of clinically significant incidental findings and the lack of need for postcontrast imaging for the detection of these incidental findings. Further, the study shows a relatively high sensitivity of the cisternographic sequence for the detection of intralesional cystic components.

Based on the results of this study, we advocate for a noncontrast MRI protocol including the acquisition of an MR cisternographic sequence for the routine surveillance of vestibular schwannomas. The ability to shift to a surveillance protocol without the administration of intravenous MR contrast is clinically relevant, given recent concerns related to repeat administration of gadolinium-based contrast agents and reported deposition of gadolinium contrast in the brain parenchyma. ${ }^{5,11,14,21,24}$ This is of particular concern in patients with vestibular schwannoma, given that surveillance examinations are routinely performed annually or biannually in this population. ${ }^{6,9,16}$ Additionally, in a metric of decreasing scan time and healthcare costs, the acquisition of a shorter and more cost-effective noncontrast MRI sequence while maintaining an accurate assessment of lesion size and internal characteristics is of paramount importance., ${ }^{2,17,22}$ At our institution, avoiding postcontrast imaging shortens the MRI acquisition time by approximately 8 minutes, eliminates the need for IV catheter placement prior to the study acquisition, and obviates the need for a serum laboratory workup to determine if a contrast agent may be administered.

In this study we looked at specific features of vestibular schwannomas, including size and internal signal characteristics. Each vestibular schwannoma was assessed based on images obtained during a single imaging session. We did not investigate recurrent vestibular schwannomas or interval growth of vestibular schwannomas. We looked at the presence of intralesional cystic components and intralesional hemorrhage as these have been reported as independent factors predicting the risk of rapid tumor growth and risk stratification for operative management of vestibular schwannomas. ${ }^{2-4,8-10,12,17,18,20,22}$ There may be additional imaging features which impact clinical management that are not addressed in this study. Although this study was conducted in a large cohort of patients with vestibular schwannomas, the number of schwannomas that demonstrated intralesional hemorrhage was infrequent (5 patients). The cohort of patients included in 

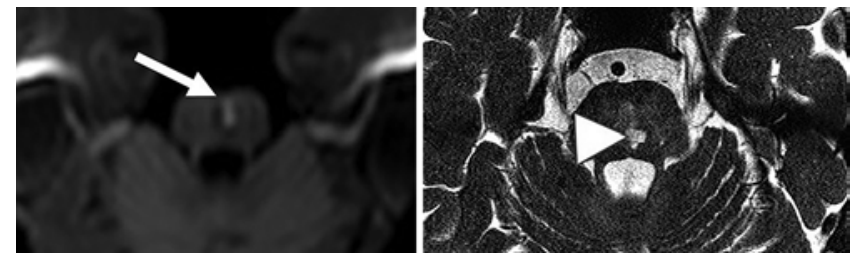

FIG. 3. Example of an incidental finding that was given a clinical significance score of 3. A 64-year-old woman was found to have an acute pontine infarct (white arrow) on the diffusion-weighted sequence (left), adjacent to a remote lacunar infarct (white arrowhead), as shown on the MR cisternographic image (right).

this study had imaging performed on both $1.5-\mathrm{T}$ and 3-T scanner platforms. While having a heterogeneous scanner platform is not ideal, the focus of this study was to evaluate various imaging characteristics of vestibular schwannomas as assessed on the MR cisternogram image compared to the postcontrast sequence acquired in the same patient. Therefore, for each patient, the MR cisternogram sequence and the postcontrast sequence were performed at the same time on the same scanner platform. A single, long-axis measurement of the vestibular schwannomas was performed in each case. While a 3D volumetric measurement may be more accurate, prior studies evaluating vestibular schwannoma size have used linear measurements. ${ }^{19}$ Lastly, the results of this study pertain only to vestibular schwannomas within the internal auditory canal and cerebellopontine angle cisterns. We did not include intralabyrinthine vestibular schwannomas in this cohort as they constitute a rare presentation. ${ }^{13,25}$

\section{Conclusions}

The results of this study demonstrated no advantage of a postcontrast MRI protocol over a noncontrast MRI protocol that includes the acquisition of an MR cisternographic sequence for the evaluation of vestibular schwannoma size and internal cystic and hemorrhagic components. Furthermore, the results of this study suggest that no potentially clinically significant incidental findings would be missed with the elimination of a postcontrast sequence. Based on the results of this study, we recommend a noncontrast MR protocol with the inclusion of a cisternographic sequence for the serial follow-up evaluation of vestibular schwannomas, sparing these patients multiple doses of gadolinium contrast over the years.

\section{References}

1. Abele TA, Besachio DA, Quigley EP, Gurgel RK, Shelton C, Harnsberger HR, et al: Diagnostic accuracy of screening MR imaging using unenhanced axial CISS and coronal T2WI for detection of small internal auditory canal lesions. AJNR Am J Neuroradiol 35:2366-2370, 2014

2. Allen RW, Harnsberger HR, Shelton C, King B, Bell DA, Miller R, et al: Low-cost high-resolution fast spin-echo MR of acoustic schwannoma: an alternative to enhanced conventional spin-echo MR? AJNR Am J Neuroradiol 17:12051210, 1996

3. Charabi S, Klinken L, Tos M, Thomsen J: Histopathology and growth pattern of cystic acoustic neuromas. Laryngoscope 104:1348-1352, 1994
4. Charabi S, Mantoni M, Tos M, Thomsen J: Cystic vestibular schwannomas: neuroimaging and growth rate. J Laryngol Otol 108:375-379, 1994

5. de Vries M, Hogendoorn PC, Briaire-de Bruyn I, Malessy MJ, van der Mey AG: Intratumoral hemorrhage, vessel density, and the inflammatory reaction contribute to volume increase of sporadic vestibular schwannomas. Virchows Arch 460:629-636, 2012

6. Fayad JN, Semaan MT, Lin J, Berliner KI, Brackmann DE: Conservative management of vestibular schwannoma: expectations based on the length of the observation period. Otol Neurotol 35:1258-1265, 2014

7. Fortnum H, O'Neill C, Taylor R, Lenthall R, Nikolopoulos T, Lightfoot $\mathrm{G}$, et al: The role of magnetic resonance imaging in the identification of suspected acoustic neuroma: a systematic review of clinical and cost effectiveness and natural history. Health Technol Assess 13:iii-iv, ix-xi, 1-154, 2009

8. Gomez-Brouchet A, Delisle MB, Cognard C, Bonafe A, Charlet JP, Deguine O, et al: Vestibular schwannomas: correlations between magnetic resonance imaging and histopathologic appearance. Otol Neurotol 22:79-86, 2001

9. González-Orús Álvarez-Morujo RJ, Alvarez-Palacios I, Martin-Oviedo C, Scola-Yurrita B, Arístegui-Ruiz MÁ: Conservative management of vestibular schwannoma. Acta Otorrinolaringol Esp 65:275-282, 2014

10. Hinoda T, Fushimi Y, Okada T, Arakawa Y, Liu C, Yamamoto A, et al: Quantitative assessment of gadolinium deposition in dentate nucleus using quantitative susceptibility mapping. J Magn Reson Imaging 45:1352-1358, 2017

11. Kanda T, Nakai Y, Oba H, Toyoda K, Kitajima K, Furui S: Gadolinium deposition in the brain. Magn Reson Imaging 34:1346-1350, 2016

12. Kandathil CK, Cunnane ME, McKenna MJ, Curtin HD, Stankovic KM: Correlation between aspirin intake and reduced growth of human vestibular schwannoma: volumetric analysis. Otol Neurotol 37:1428-1434, 2016

13. Kennedy RJ, Shelton C, Salzman KL, Davidson HC, Harnsberger HR: Intralabyrinthine schwannomas: diagnosis, management, and a new classification system. Otol Neurotol 25:160-167, 2004

14. Kuno H, Jara H, Buch K, Qureshi MM, Chapman MN, Sakai O: Global and regional brain assessment with quantitative MR imaging in patients with prior exposure to linear gadolinium-based contrast agents. Radiology 283:195-204, 2017

15. Martin TP, Senthil L, Chavda SV, Walsh R, Irving RM: A protocol for the conservative management of vestibular schwannomas. Otol Neurotol 30:381-385, 2009

16. Matthies C, Samii M, Krebs S: Management of vestibular schwannomas (acoustic neuromas): radiological features in 202 cases - their value for diagnosis and their predictive importance. Neurosurgery 40:469-482, 1997

17. Mehrotra N, Behari S, Pal L, Banerji D, Sahu RN, Jain VK: Giant vestibular schwannomas: focusing on the differences between the solid and the cystic variants. Br J Neurosurg 22:550-556, 2008

18. Nutik SL, Babb MJ: Determinants of tumor size and growth in vestibular schwannomas. J Neurosurg 94:922-926, 2001

19. Ozgen B, Oguz B, Dolgun A: Diagnostic accuracy of the constructive interference in steady state sequence alone for follow-up imaging of vestibular schwannomas. AJNR Am J Neuroradiol 30:985-991, 2009

20. Park CK, Kim DC, Park SH, Kim JE, Paek SH, Kim DG, et al: Microhemorrhage, a possible mechanism for cyst formation in vestibular schwannomas. J Neurosurg 105:576-580, 2006

21. Reeder SB, Gulani V: Gadolinium deposition in the brain: do we know enough to change practice? Radiology 279:323326, 2016

22. Schmidt RF, Boghani Z, Choudhry OJ, Eloy JA, Jyung RW, 
Liu JK: Incidental vestibular schwannomas: a review of prevalence, growth rate, and management challenges. Neurosurg Focus 33(3): E4, 2012

23. Sidman JD, Carrasco VN, Whaley RA, Pillsbury HC III: Gadolinium. The new gold standard for diagnosing cerebellopontine angle tumors. Arch Otolaryngol Head Neck Surg 115:1244-1247, 1989

24. Stojanov D, Aracki-Trenkic A, Benedeto-Stojanov D: Gadolinium deposition within the dentate nucleus and globus pallidus after repeated administrations of gadolinium-based contrast agents-current status. Neuroradiology 58:433-441, 2016

25. Zhu AF, McKinnon BJ: Transcanal surgical excision of an intracochlear schwannoma. Am J Otolaryngol 33:779-781, 2012

\section{Disclosures}

The authors report no conflict of interest concerning the materi- als or methods used in this study or the findings specified in this paper.

\section{Author Contributions}

Conception and design: Buch, Cunnane, Acquisition of data: Buch, Juliano, Cunnane. Analysis and interpretation of data: all authors. Drafting the article: Buch, Stankovic, Curtin, Cunnane. Critically revising the article: all authors. Reviewed submitted version of manuscript: all authors. Approved the final version of the manuscript on behalf of all authors: Buch. Statistical analysis: Buch, Juliano, Cunnane. Administrative/technical/material support: Curtin. Study supervision: Curtin, Cunnane.

\section{Correspondence}

Karen Buch: Massachusetts General Hospital, Boston, MA. karen. buch@gmail.com; kbuch@partners.org. 